\title{
How to Facilitate Organizational Transformation with a Turnaround Mindset and an Innovation Culture
}

\author{
Mark Faust
}

Received: 6 July 2013 / Accepted: 2 August 2013 / Published online: 26 October 2013

(C) Springer Science+Business Media Dordrecht 2013

\begin{abstract}
If all the stars were to align, and if innovations yet to be thought of were implemented, how much more do you think that you and your team as a whole could realistically create in annual sales revenue and profits? I believe and have experienced that the vast majority of companies have the potential to dramatically accelerate growth. With aggressive innovation and effective strategy, three-year objectives can often become this year's reality. However, companies achieve this not from efforts focused just on marketing or individual selling skills or productivity innovations for growth, but by focusing holistically throughout a company. If a concerted effort is made to accelerate growth from every angle possible, often significant growth is right around the corner. The methods below are widely applicable to the silicon-based industry and the end users of silicon-based products.
\end{abstract}

Keywords Turnaround · Innovation · Growth . Productivity

\section{Introduction}

Improving sales and profits has little to do with sales training, consulting, or other quick fixes. I've experienced that most sales-people and teams are already gifted with enough ideas and ability to foster significant increases in sales and profits, but they are often harnessed by procedures that limit productivity or approaches that constrain the level of

\section{Faust ( $\square)$}

Echelon Management,

8044 Montgomery Road, \#260, Cincinnati, OH 45236, USA

e-mail: Mark@em1990.com

URL: www.EchelonManagement.com idea exchange and customer engagement. Fostering new growth is more about innovation than marketing angles, sales productivity, or skills; growth is more about a culture of continuous improvement than marketing blitz; growth is more about possibility-thinking than fear-fostering quotas. Your job is to prioritize a few new steps and ideas that can be implemented in a reasonable time. To grow your business, you must become the facilitator of holistic innovation and a growth revolution [1-3].

\section{Don't Just Choose to Take a Step Forward and Choose to "Turnaround"}

\subsection{The Revolution Solution}

Whether your company's growth rate is not what it could be or its very existence is at risk, your leadership team would do well to take the attitude of facilitating a turnaround. I have seen the best results when the CEO or other top leader declared boldly across the company, "We are about to embark on a turnaround!" Or, "As well as we are doing right now, I know that, with the talent and potential in this organization, we are capable of much greater things; we need to have an attitude that we are embarking on an innovation and growth revolution". This attitude of "growth regardless of current success" is one aspect of The Revolution Solution (see Fig. 1). A determining factor of any possible growth is a leader's belief in what is possible for his or her organization.

The alternative to a turnaround or revolution mentality is, "Let's tweak and improve a few things just enough that we don't upset what's working but achieve some improved profitability". Your team will see it as just another management flavor of the month. Too often leaders who approach 
Fig. 1 The revolution solution mentality (see Ref. [1] p 28)

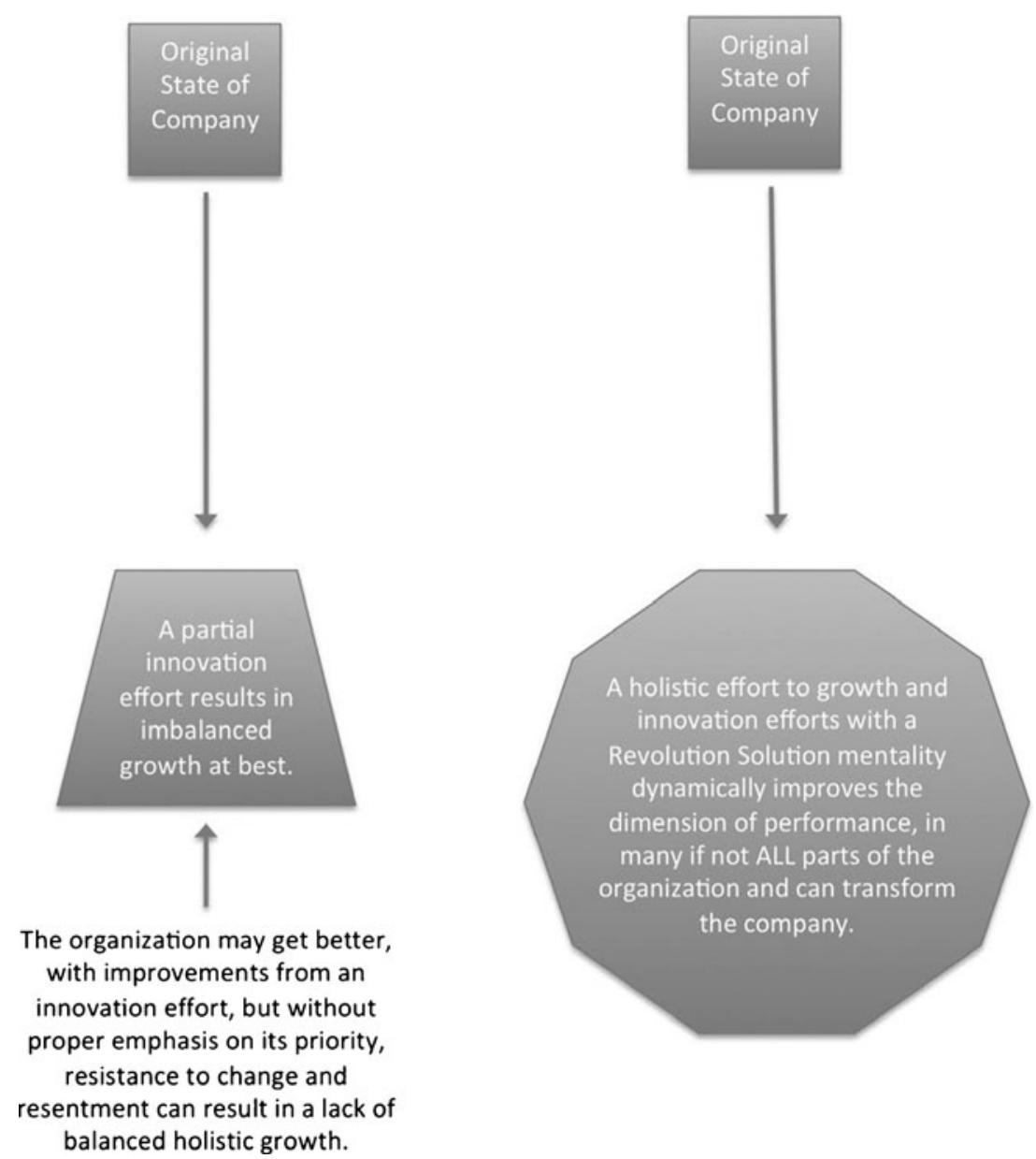

growth this way are only looking at a couple of areas of improvement. For example, they might look at how they could increase sales and decrease production costs. The result of taking this piecemeal approach to improvement is that there will most likely be pushback from the one or two areas you've decided to improve. Production will say, "What about sales?!" Or sales will resent that you are focusing on them while marketing is slacking in coming up with the new tools or the new leads they have been begging for.

Your attitude, approach, and aim for areas in need of improvement must be holistic. Innovation can come in every area of any organization, and merely pointing at a few particular areas and omitting others from the improvement process will breed bitterness, resentment, and efforts to hinder success and blame others.

\subsection{The Benefits of a Turnaround Mindset}

Whether your company is behind the eight ball with the clock ticking towards bankruptcy or there is little risk to your existence, I exhort your leadership to decide (and then communicate to the company) "We are in turnaround mode" (see Fig. 2). The statement adds a healthy amount of drama and emphasizes the significance of your new focus. Explain as well that dramatic cost-cutting is about to begin, but this does not mean people must be laid off.

Too often these types of slash-and-burn techniques are the tools of lazy, inept, uncreative, and unqualified

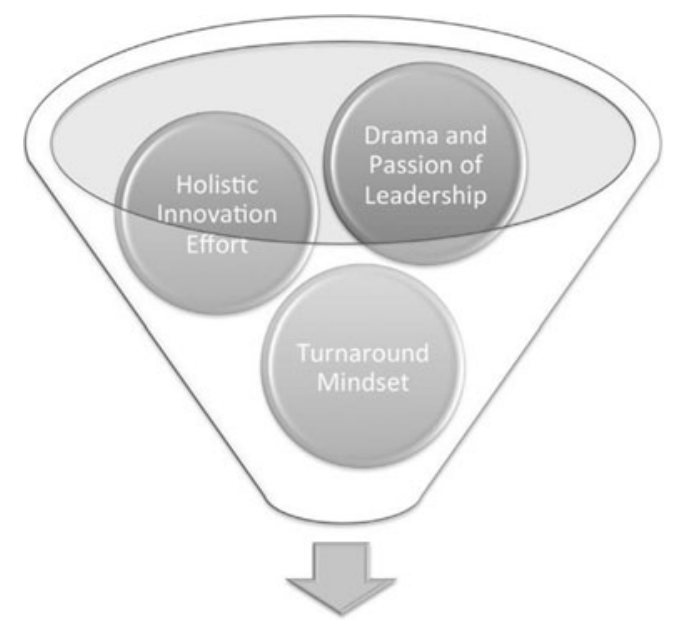

Fig. 2 The turnaround mindset (see Ref. [1] p 29) 
leadership and consultants who are taking the easy way out of a challenging situation. Sadly, the idea of dramatically increasing profits through innovation and growth has become revolutionary rather than the norm.

\subsection{Innovation is the Root of all Growth and Every Turnaround}

The two core functions of a business are innovation and marketing. The two are inextricably connected, and your team must understand what this means for your business and how they as team members must contribute. They must know that innovation doesn't only apply to product improvements, but to service and even internal process improvement as well. Most especially it needs to apply to all elements that effect the growth of revenue and profitability.

At our client companies where we have facilitated the innovation process, the impact is consistently significant; seeing millions in potential profit improvement is not uncommon for small and mid-sized companies. Ensuring that your company is innovating in an ongoing process is guaranteed to produce significant rewards in your growth and bottom line.

\section{The 7 Facets of Building an Innovation Culture}

A corporate culture that is not fertile to cultivate such growth will quell profit-building innovation efforts. This is why you must often start with turning around your culture in order to make your team's hearts, minds, and hands work for an environment of growth-focused innovation.

\subsection{Prioritize from the Top Down}

The foundation for a culture of accelerated innovation is the titular leader's firm resolution and well-publicized commitment that innovation is a top priority. The top dog must consistently communicate this focus and motivate the team with it.

\subsection{Clarify Your Innovation Values}

Employees will want a values list to include things like respect and honesty, but the top leader needs to insure that those values are included that are key to the innovation's success. Humility is a foundational value, as it requires everyone to admit that there could always be a better way, and that others may have better insight or answers. Open communication and safety in communicating problems are critical values, as people will clam up if an authority figure pounces on their suggestions for areas to improve.
To ensure that innovation at your company will be ongoing and not just a temporary spurt, you should build innovation not only into the company values, but also into the growth objectives and the mission and vision as well.

\subsection{Get All Hands on Deck}

Every facet of the business can be improved and every person must be committed to making regular efforts for improvement. Every person is exposed to at least a few areas of the business where they must be asked to think about what could be better. Management must require a minimum number of ideas to be turned in from every employee.

\subsection{Initially Focus on Quantity vs. Quality}

A key to success in innovation is generating the optimum quantity of ideas, it is better to have created 1,000 new ideas (regardless of whether they are mediocre or impractical) than only a handful of excellent ideas in your initial efforts. Research consistently proves that a quantity of ideas will beget better-quality ideas in the end (see Fig. 3). This is because the multitude of ideas will evolve and ultimately lead to the most valuable ideas for implementation.

\subsection{Consistently Communicate Implementation and Successes}

Getting everyone to begin looking for improvement opportunities and solutions for implementation changes the thinking and habits of the team. To continue the growth of such habits, employees must have a workplace environment with continuous feedback. Employees must regularly hear how their ideas and everyone's participation in innovation are improving their business with as many

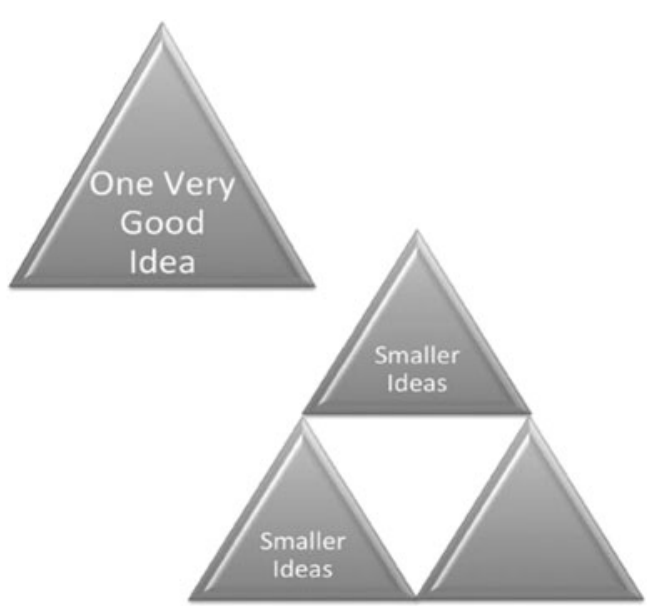

Fig. 3 A quantity of ideas leads to better and bigger ideas (see Ref. [1] p 34) 


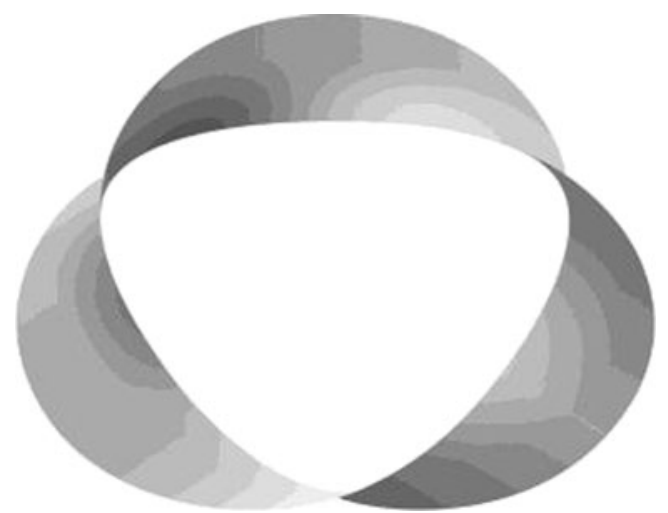

Fig. 4 Like a Möbius loop, innovation efforts need to be ongoing (see Ref. [1] p 35)

specific reports of improvements and implemented changes as possible.

\subsection{Give Specific and Universal Rewards}

Management may lament that it's everyone's job to keep an eye out for what can be improved, but the fact is that strategically recognized and incentivized good behaviors become repeated and habitual. Individuals who recommend ideas that have significant impact to profits should be given significant rewards. As the tide of innovation risesa tide that is the result of a team contributing great efforts toward innovation-monetary and other rewards should be shared with the team. Innovation pays for itself and is self-perpetuating.

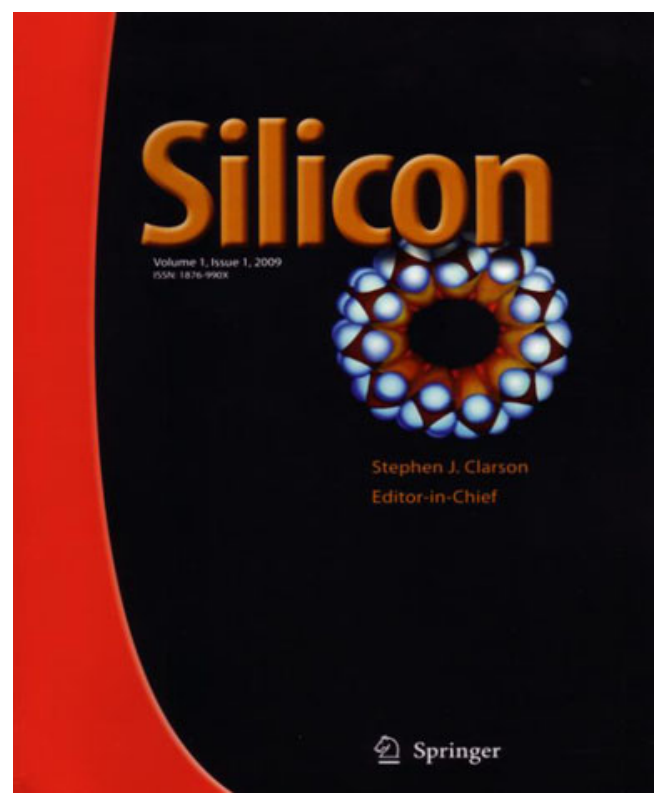

Fig. 5 The siloxane $\mathrm{D}_{22}$ ring as shown on the cover of SILICON. It is never-ending, consistent, and based on the single plane of an innovation-based, values-oriented culture

\subsection{Make Innovation a Möbius Strip}

Leading and managing the innovation process and culture takes work, and thus when times are good some may want to rest and ride out their competitive advantages. However, innovation must be an ongoing process at sustainable levels. Avoid innovation efforts in spurts as this causes long-term employees to dub any new efforts the "management flavor of the day". The process must be more like the path of a Möbius strip (see Fig. 4) or a the path round a cyclic molecule (see Fig. 5) [4].

\section{Concluding Remarks}

In conclusion it is important to remember that every organization needs innovation objectives. These must be specific and measurable targets against which you can measure your innovation efforts both in quantity and quality. As you venture to raise the bar of innovation in your organization, you also must wield the strategic abandonment sword. Innovation is as much about what you stop doing as what you begin.

As the late Steve Jobs pointed out:

"If you want to live your life in a creative way, as an artist, you have to not look back too much. You have to be willing to take whatever you've done and whoever you were and throw them away". (Playboy, 1985).

"People think focus means saying yes to the thing you've got to focus on. But that's not what it means at all. It means saying no to the hundred other good ideas that there are. You have to pick carefully. I'm actually as proud of the things we haven't done as the things I have done. Innovation is saying no to 1,000 things". (Apple Worldwide Developers' Conference, 1997)

Acknowledgments We must acknowledge Peter Drucker who was the first to effectively demystify innovation and detail how it is a repeatable science and not a gift that some are given and others are not. It was he who pointed out so effectively that it is both a responsibility and requirement fore the effective managers of any institution that is determined to thrive.

\section{References}

1. Faust M (2011) Growth or bust! Proven turnaround strategies to grow your business. Career Press, Pompton Plains, NJ

2. Mark Faust is a growth advisor, a professional speaker and the founder of Echelon Management a growth and turnaround consultancy. Contact him at Email: Mark@em1990.com or Tel.: 513.621 .8000

3. Growth tweets are available from @MarkFaustSr

4. Clarson SJ (2009) Silicon 1:1-2. doi:10.1007/s12633-009-9006-2 